\title{
Fatty acid kinase $A$ is an important determinant of biofilm formation in Staphylococcus aureus USA300
}

\author{
J. S. Sabirova ${ }^{1,2}$, J-P Hernalsteens², S. De Backer ${ }^{1}$, B. B. Xavier ${ }^{1}$, P. Moons ${ }^{1}$, A. Turlej-Rogacka ${ }^{1}$, H. De Greve ${ }^{3,4}$, \\ H. Goossens ${ }^{1}$ and S. Malhotra-Kumar ${ }^{5^{*}}$
}

\begin{abstract}
Background: Methicillin-resistant Staphylococcus aureus (MRSA)-USA300 is notorious for its ability to cause community- and healthcare-acquired infections, which are even more difficult to treat when associated with a biofilm phenotype. We aimed to characterize the genetic determinants of biofilm formation in a USA300 skin abscess isolate (UAS391) that formed prolific biofilms.

Methods: USA300 S. aureus strains, TCH1516 and FPR3757, were found to be closely related based on whole genome mapping (Argus ${ }^{\text {TM }}$ Optical Mapping System, Opgen Inc, Gaithersburg, USA) to UAS391 (96.3-99.1 \% similarity, $P=0.0151$ ), however differed markedly in biofilm formation ( $P=0.0001$ ) on a dynamic assay (BioFlux 200, Fluxion Biosciences, USA). Comparison of whole genome sequences of these strains identified differences in a total of 18 genes. Corresponding Tn (bursa aurealis-bearing) knockout mutants in these target genes were obtained from a publicly available mutant library of the same clonal lineage (USA300-JE2) and were characterized phenotypically for biofilm formation. Tn mutants showing significant differences in biofilm formation were utilized for transduction into a plasmid-cured erythromycin-sensitive derivative of UAS391 and for complementation experiments. All strains were tested on the dynamic assay, and 17h-biofilms were stained (SYTO9, Life Technologies) and fluorescence intensity quantified by microscopy (Zeiss, ImageJ). Gene expression levels in Tn and transduced mutants were studied by quantitative reverse transcriptase PCR (StepOnePlusTM, Applied Biosystems ${ }^{\circledR}$ ).
\end{abstract}

Results: Comparison of the sequenced genomes of TCH1516, FPR3757 and UAS391 yielded a limited number of variant genes $(n=18)$ that were hypothesized to account for the observed difference in biofilm-forming capacity. Screening of Tn mutants disrupted in these target genes identified one mutant (NE229) bearing a transposon insertion in SAUSA300_1119 (fakA), which exhibited increased biofilm formation similar to UAS391 ( $\mathrm{P}=0.9320)$. Transduction experiments confirmed that fakA::Tn corresponded to 1.9- to 4.6-fold increase in biofilm formation depending on the USA300 strain background (PS0.0007), while complementation of the TCH1516 wild-type fakA allele in UAS391 resulted in a 4.3-fold reduction in biofilm formation $(P<0.0001)$.

Conclusions: This sequential approach, consisting of strain typing, genome comparison and functional genomics, identified fakA, a recently described fatty acid kinase in $S$. aureus that is essential for phospholipid synthesis and also impacts the transcription of numerous virulence factors, as a negative regulator of biofilm formation in S. aureus USA300.

Keywords: vfrB, fakA, MRSA, Methicillin-resistant S. aureus, Transposon mutagenesis, Whole genome sequencing, SNPs, USA300-UAS391, USA300_UAS391, USA300, UAS391

\footnotetext{
* Correspondence: surbhi.malhotra@uantwerpen.be

Part of this work was presented at the European Congress of Clinical

Microbiology and Infectious Diseases 2013 (ECCMID).

${ }^{5}$ Department of Medical Microbiology, Campus Drie Eiken, University of

Antwerp, S6, Universiteitsplein 1, B-2610 Wilrijk, Belgium

Full list of author information is available at the end of the article
}

(c) 2015 Sabirova et al. Open Access This article is distributed under the terms of the Creative Commons Attribution 4.0 International License (http://creativecommons.org/licenses/by/4.0/), which permits unrestricted use, distribution, and reproduction in any medium, provided you give appropriate credit to the original author(s) and the source, provide a link to the Creative Commons license, and indicate if changes were made. The Creative Commons Public Domain Dedication waiver (http://creativecommons.org/publicdomain/zero/1.0/) applies to the data made available in this article, unless otherwise stated. 


\section{Background}

Staphylococcus aureus causes infections ranging from minor skin infections to life-threatening diseases, such as pneumonia, meningitis, osteomyelitis, endocarditis, and septicaemia. Since their emergence in the 1960s, methicillin-resistant $S$. aureus have become one of the major causes of hospital-acquired (HA) infections such as implant-associated and postsurgical wound infections, as well as of community-acquired (CA) infections such as pneumonia. The success of these hospital-acquired MRSA (HA-MRSA) clones can be partly attributed to virulence-specific factors, such as extracellular toxins, surface structures facilitating tissue colonization, immune evasion and tissue destruction [1], as well as to prolonged persistence of MRSA infections linked to the formation of biofilms in vivo [2].

It is generally accepted that biofilms, comprising conglomerations of cells attached to a solid support and embedded in a matrix of extracellular polymers, represent a major problem in clinical practice, due to their formation on implanted medical devices [3] and their intrinsic enhanced resistance to antibiotics that are otherwise efficacious against the bacterium's planktonic life forms [4]. These biofilm-associated complications have triggered the search for potential genes and/or metabolic pathways, interruption of which could represent new therapeutic or preventive interventions specifically targeting this bacterial life-style. Functional genomics approaches such as transcriptomics and proteomics, performed on biofilm versus planktonic cells, have shed some light on the complexity of the biofilm phenotype in S. aureus. Among genes found to be up-regulated in biofilm cells were those encoding proteins involved in the synthesis of polysaccharide intercellular adhesin (PIA)/ polymeric $N$-acetyl-glucosamine (PNAG) as well as proteins mediating transport, amino acid metabolism and translation, with many other up-regulated genes encoding hypothetical proteins of still unknown function [5]. Subsequent proteomic studies performed by Resch et al. [6] showed still more stringently that biofilm-induced proteins are involved in cell attachment and peptidoglycan synthesis, in pyruvate and formate metabolism, as well as in regulatory processes, in particular those exerted by the staphylococcal accessory regulator A protein.

We recently screened a large collection of clinically important MRSA isolates for their ability to form biofilms, and subsequently typed these isolates [7]. This work yielded a number of strong biofilm-forming strains, with three of them belonging to the USA300 clonal lineage, one of which was selected for genome sequencing. Subsequent comparison of the strain's genome with those of other USA300 sequenced isolates revealed a particularly interesting pair of closely related USA300 strains showing notably different capacities for biofilm formation (UAS391 and USA300_TCH516). This finding offered the opportunity to search in clinical isolates at genome level for mechanisms of biofilm formation. Thus, various USA300 S. aureus clones, which are closely related as per whole genome mapping, but markedly different in biofilm formation, were compared at genome level and consequently genetic loci with different alleles were identified. Corresponding knockout mutants of these genes, obtained from a publically available mutant library of the same clonal lineage (USA300-JE2), were then phenotypically characterized for their potential role in biofilm formation. Transposon-mediated interruption of one of the tested divergent genes, SAUSA300_1119, corresponding to the gene fakA, resulted in drastically increased levels of biofilms as compared to the parental control strain JE2, marking this gene as an important determinant of biofilm formation. These results shed more light on the genetic factors regulating biofilm formation in $S$. aureus.

\section{Methods}

\section{Bacterial strains and growth conditions}

USA300 strains used for clonal, genomic, and phenotypic analyses are listed in Table 1 . Tn insertion mutants used for genotypic and phenotypic analysis of biofilm formation, were obtained from a sequence-defined transposon mutant library consisting of 1,952 strains, each containing a single mutation within a nonessential gene in strain USA300 JE2 [8] obtained from the NARSA repository (www.beiresources.org/). S. aureus USA300 FPR3757 (FPR3757) and USA300 JE2 (JE2) were also obtained from the NARSA repository. Strains UAS391, TCH1516, FPR3757 and JE2 were routinely grown on brain heart infusion (BHI) and lysogeny broth (LB) medium for biofilm and transduction experiments, respectively. Transposon insertion mutants were grown on BHI medium containing $10 \mu \mathrm{g} / \mathrm{ml}$ of erythromycin (Sigma-Aldrich).

\section{Transduction experiments}

Transduction was performed essentially as described [9]. Transducing phage $\phi 11$ was obtained from the supernatant of a culture of the lysogenic $S$. aureus strain RN0451 (NARSA strain NRS136) and propagated on $S$. aureus RN0450 (NARSA strain NRS135) by standard techniques [10]. A transducing phage stock was prepared by infection at $37{ }^{\circ} \mathrm{C}$ of $S$. aureus containing the bursa aurealis transposon insertion. After infection of cultures of the recipient S. aureus strains (UAS391 and TCH1516) with this stock, transductants were selected on LB plates with $0.05 \%$ sodium citrate containing $5 \mathrm{mg} / \mathrm{L}$ erythromycin at $37{ }^{\circ} \mathrm{C}$. The resulting colonies were purified at least twice on the same medium to ensure loss of the transducing phage. 
Table 1 Strains and plasmids used for clonal, genomic, and phenotypic analyses during this study

\begin{tabular}{|c|c|c|}
\hline Strains/Plasmids & Description & Source \\
\hline \multicolumn{3}{|l|}{ Strains } \\
\hline UA S391 & S. aureus strain USA_UAS391 & [18] \\
\hline JE2 & S. aureus USA300 parental strain for the NARSA transpos on library & NARSA ${ }^{a}$ \\
\hline FPR3757 & S. aureus USA300_FPR3757 & NARSA \\
\hline TCH1516 & S. aureus subsp. aureus USA300_TCH1516 & ATCC \\
\hline UA S391-Ery ${ }^{5}$ & Heat-cured erythromycin-sensitive derive of UAS391 & This study \\
\hline TCH1516-Ery ${ }^{5}$ & Heat-cured erythromycin-sensitive derive of TCH1516 & This study \\
\hline UA S391-NE229 & UA S391-Ery ${ }^{5}$ transductant with mutation in EX97_05885 & This study \\
\hline TCH1516-NE229 & TCH1516-Ery ${ }^{5}$ transductant with mutation in USA 300Hou_1162 & This study \\
\hline NE229-pHD954 & S. aureus stra NE229 completed with pHD954 & This study \\
\hline NE229-pHD957 & S. aureus strain NE229 completed with pHD957 & This study \\
\hline UA S391-pHD957 & S. aureus strain UA S391 completed with pHD957 & This study \\
\hline RN0451 & Phage \$11 lysogenic S. aureus strain (NARSA strain NRS 136) & NARSA \\
\hline RN0450 & S. aureus RN0450 (NARSA strain NRS 135) & NARSA \\
\hline \multirow[t]{2}{*}{ RN4220 } & Restriction-deficient intermediate S. aureus cloning host RN4420 & NARSA \\
\hline & Restriction-deficient intermediate S. aureus cloning host RN4420 & NARSA \\
\hline NE1646 & Transposon mutant with insertion in USA200HOU_0155 (SA USA300_0145) & NARSA \\
\hline NE229 & Transpos on mutant with insertion in USA300HOU_1162 (SA USA300_1119) & NARSA \\
\hline NE1081 & Transpo on mutant with insertion in USA300HOU_2626 (SA USA300_2561) & NARSA \\
\hline NE454 & Transpo on mutant with insertion in USA300HOU_2631 (SA USA300_2566) & NARSA \\
\hline NE1290 & Transposon mutant with insertion USA300HOU_2641 (SA USA300_2576) & NARSA \\
\hline NE81 & Transposon mutant with insertion in USA300HOU_2319 (SA USA300_2285) & NARSA \\
\hline NE1036 & Transposon mutant with insertion in USA300HOU_2602 (SA USA300_2542) & NARSA \\
\hline NE1038 & Transposon mutant with insertion in USA300HOU_2678 (SA USA300_2610) & NARSA \\
\hline NE33 & Transposon mutant with insertion in USA300HOU_2654 (SA USA300_2589) & NARSA \\
\hline NE1262 & Transposon mutant with insertion in USA300HOU_2026 (SA USA300_1984) & NARSA \\
\hline NE1875 & Transposon mutant with insertion in USA300HOU_1934 (SA USA300_1918) & NARSA \\
\hline NE1 & Transposon mutant with insertion in USA300HOU_1372 (SaA USA300_1327) & NARSA \\
\hline NE334 & Transposon mutant with insertion in USA300HOU_2197 (SA USA300_2161) & NARSA \\
\hline NE809 & Transposon mutant with insertion in USA300HOU_1260 (SA USA300_1214) & NARSA \\
\hline NE1403 & Transposon mutant with insertion in USA300HOU_1260 (SA USA300_1585) & NARSA \\
\hline NE1026 & Transposon mutant with insertion in USA 300HOU_1338 (SA USA 300_1298) & NARSA \\
\hline NE1314 & Transposon mutant with insertion in USA300HOU_0953(SA USA300_0896) & NARSA \\
\hline \multicolumn{3}{|l|}{ Plasmids } \\
\hline pALC2073 & Shuttle vector pALC2073 & [11] \\
\hline pHD954 & pALC2073 with cloned fakA gene amplifie from UAS391 & This study \\
\hline pHD957 & pALC2073 with cloned fakA gene amplifie from TCH15116 & This study \\
\hline
\end{tabular}

${ }^{\text {aTh }}$ library now exists at www.beiresources.org and is discussed by Fey et al. [8]

\section{Complementation experiments}

To complement the $S$. aureus mutant strain NE229, total genomic DNA of strains UAS391 and TCH1516 was purified with the Quick Pick ${ }^{\mathrm{TM}}$ SML gDNA kit (BN Products \& Services) according to the manufacturer's recommendations. S. aureus strains were lysed by adding $5 \mu \mathrm{g}$ lysostaphin (Sigma-Aldrich). The genes corresponding to
SAUSA300_1119 from the S. aureus strains UAS391 and TCH1516 were amplified using ExTaq DNA polymerase (Takara) with the primer pair Glyk-1 (5'-TACCGAGCTCGAATTCTAGGAGGACAACTTGAAATGATTAG-3') and Glyk-2 (5'-GACGGCCAGTGAATTCATTTTTATTCTACTGAAAAGAAATATTG-3'). Polymerase chain reactions (PCR) were carried out in an Applied Biosystems 2720 
Thermal Cycler using Ex Takara DNA Polymerase. Annealing and elongation temperatures were $55{ }^{\circ} \mathrm{C}$ and $68{ }^{\circ} \mathrm{C}$ respectively, with an elongation time of 1 min per $1000 \mathrm{bp}$. during 30 cycles. PCR-fragments were purified using the Qiaquick PCR Purification Kit (Qiagen $\mathrm{GmbH}$ ) and analysed by gel electrophoresis on $1.0 \%$ agarose gels. The resulting $1753 \mathrm{bp}$. PCR fragments were cloned by the InFusion technique (Clontech Laboratories, Inc) in the EcoRI site of the shuttle vector pALC2073 [11] yielding the plasmids pHD954 (UAS391) and pHD957 (TCH1516). DNA sequencing was performed at the VIB core sequencing facility (VIB Genetics Department, University of Antwerp) using the pALC2073 vector primers TetR2 (5'-CAATGTAGGCTGCTCTACACCTAG-3'), pALC-2 (5'GATCGGTGCGGGCCTCTTCGCTAT-3'), and the internal gene sequence primers Glyk-3 (5'-GGAGTACATTATTGTAAAAGCCAATGAATC-3') and Glyk-4 (5'- CCACACATATCATTAGTGGTGGACA-3'). These plasmids were transferred into the restriction-deficient intermediate $S$. aureus cloning host RN4220 to adapt the plasmid DNA [12] to the $S$. aureus modifications. Transformants were selected on LB plates supplemented with $10 \mu \mathrm{g} / \mathrm{ml}$ chloramphenicol (Sigma-Aldrich). The plasmids pHD954 and pHD957 were isolated from the RN4220 strain and used to transform strain NE229. UAS391 was also complemented with the wild-type fakA allele carried on pHD957.

\section{RNA extraction and RT-PCR}

Mutant strain NE229 and wild-type strains FPR3757 and JE2 were grown as overnight cultures in $10 \mathrm{ml}$ of BHI medium at $37{ }^{\circ} \mathrm{C}$ with shaking. Total RNA of the three bacterial strains was extracted after $16 \mathrm{~h}$ growth using Express Amptec kit (Ambion). Two $\mu$ g of RNA was treated with DNase using the Turbo DNA-free ${ }^{\mathrm{rm}} \mathrm{Kit}$ (Ambion ${ }^{\circ}$ by Life Technologies ${ }^{\mathrm{Tx}}$ ) and subsequently used for reverse transcription reaction using the Reverse Transcription System (Promega) with random primers according to the manufacturer's instructions. Real-time PCR was performed using a StepOnePlus ${ }^{\text {tu }}$ system (Applied Biosystems ${ }^{\circ}$ ) in a $20 \mu \mathrm{l}$ reaction mixture with Absolute Blue qPCR SYBR green ROX mix (Thermo Scientific, Inc). For RT-PCR analysis, the cDNA samples were amplified with gene-specific primers 1162 F (5'-ATGATGTGGACGCAACACTTG-3') and 1162Rev (5'-AATCAAGCCCATAAACGCGTC-3'), in duplicate. Cycling conditions were $95^{\circ} \mathrm{C}$ for $10 \mathrm{~min}$, and 40 cycles of $95^{\circ} \mathrm{C}$ for $15 \mathrm{~s}$ and $60{ }^{\circ} \mathrm{C}$ for $60 \mathrm{~s}$ and $72{ }^{\circ} \mathrm{C}$ for $40 \mathrm{~s}$. Amplification plot and melting curve were analysed for the dynamics of fluorescence and specificity of amplification, correspondingly. Resulting PCR products were checked by gel electrophoresis.

\section{Whole genome mapping}

For WGM, UAS391 and TCH1516 were grown on BHI plates, and high molecular weight DNA was extracted from the overnight colonies using Argus ${ }^{\oplus} \mathrm{HMW}$ DNA extraction kit (Opgen, Inc). Following DNA extraction as recommended by manufactures protocol, DNA molecules were loaded on a MapCard surface (Opgen, Inc) where single DNA molecules were immobilized and linearized. The linearized DNAs were subjected to in situ digestion with NcoI (Opgen, Inc). Following digestion, the DNA molecules were stained with the fluorescent intercalating agent JoJo-1 forming part of the staining kit (Opgen, Inc). The digested and stained DNA fragments were imaged and assembled by in built assembler of Map Manager to produce whole genome restriction maps, as described previously [13]. Finally, derived whole genome maps were analysed using BioNumerics v7.5. (Applied Maths, Belgium).

\section{Genome sequencing}

The complete genome sequence of UAS391 was generated by using the Illumina HiSeq2000 platform, as described previously [14]. Sequence data of UAS391 were de novo assembled using Velvet [15] and SPAdes [16]. Assembled contigs were ordered against the UAS391 whole genome map using MapSolver software (Opgen, Inc). Validated scaffolds were ordered against published S. aureus genome TCH1516 (accession no. CP000730), and the generated pseudo chromosome was compared to the genomes of TCH1516 and FPR3757 (accession no. CP000255.1) using Mauve v2.3.1 [17]. Similarly, we also performed reference assembly independently by using the genome sequences of TCH1516 and FPR3757 as references, and SNPs were extracted in CLC Genomics Workbench 7.5.1 (QIAGEN, Aarhus A/S, Denmark). The assembled chromosome of UAS391 was annotated as described [18]. Multiple alignment and phylogenetic analysis were performed using MEGA6 [19].

\section{Flow biofilm assay and quantification of biofilm mass}

A medium-throughput continuous flow system BioFlux 200 (Fluxion Biosciences, USA) was used to study biofilm formation under shear flow conditions, which mimics flow conditions of physiological liquids in the human body [20]. BHI or $0.5 x B H I$ with $0.1 \%$ glucose was used to feed into the flow cell. Bacterial cultures at 0.05 MacFarland were then used to inoculate the output wells; bacteria were pushed through the flow cell from the output well up to the horizontal microfluidic channel by reversing the flow and were allowed to attach for one hour followed by $16 \mathrm{~h}$ of incubation at $37^{\circ} \mathrm{C}$ in BHI or $0.5 \mathrm{xBHI}$ with $0.1 \%$ glucose at a flow rate of 0.5 dyne/ $\mathrm{cm}^{2}$. Biomass in the microfluidic channels was stained with SYTO 9 fluorescent stain (Invitrogen, Life Technologies). Biofilm images were captured employing ZEN 2012 software (Zeiss) as combined tile images consisting of 81 one $\mu \mathrm{m}^{2}$ horizontal tiles covering the entire 
microchannel. Actual fluorescence quantification was recorded as integrated density on the entire combined tile image using Image J freeware (http://imagej.nih.gov/) using "integrated density", "mean value" and "area" as measurement settings. Presented values are averages of three independent combined tile images.

\section{Statistical analysis}

Statistical analysis of biomass formation in the dynamic flow assay was performed using the $\mathrm{R}$ Project software (version 3.1.2.). The influence of a particular gene interruption on biofilm formation and the comparison of the space occupied by the cells within the dynamic biofilms were analysed using a pairwise one-way ANOVA, Shapiro-Wilk normality testing, Bartlett's test of variances, and Tukey's honest significance difference testing. Results from RT-PCR were analysed through a Wilcoxon signedrank test. $P<0.05$ were considered to be significant.

\section{Results}

Typing and phenotypic analysis of USA300 isolates

Whole genome mapping (WGM) allowed typing and grouping of strain UAS391 with sepsis strain USA300_TCH1516 (TCH1516) isolated at Texas Children's Hospital in Houston [21] along with two other $S$. aureus USA300 strains, USA300-FPR3757 (FPR3757) and its plasmid-cured laboratory derivative USA300-JE2 (JE2), both belonging to the USA300 clonal lineage. According to the WGM similarity cut-off recently established for USA300 isolates [22], a WGM-based clonal cluster is defined as a set of isolates having a whole genome map similarity of $>95 \%$, which assigned all four isolates discussed here to the same WGM clonal cluster (Fig. 1). As UAS391 was previously identified as a prolific biofilm former [7], we directly compared its biofilm forming capacity to the other clonally related strains in a dynamic biofilm assay. Comparison of UAS391 with the other USA300 isolates in this dynamic biofilm model consistently revealed clear differences in this strain's ability to form biofilms under these conditions, with UAS391 forming 1.6- to 3.6-fold more biofilm than the other three USA300 strains, as measured by readout of fluorescence $(P<0.0001$, ANOVA, $\mathrm{F}=56.95, \mathrm{df}=3)($ Fig. 2$)$.

\section{Identifying potential gene targets by comparative genomics}

Genomic divergence between phenotypically diverse strains from the USA300 clonal lineage should by definition be very limited $[21,23]$. Since TCH1516 was closely related to UAS391 as per WGM and its total genome sequence is already available [21], we reasoned that comparing the whole genome sequences of UAS391 and TCH1516 would likely yield genetic differences that could account for the observed differences in the biofilm phenotype between these two USA300 strains. We thus sequenced the UAS391 genome by using the Illumina HiSeq2000 platform [18], and identified a total of 52 gene loci where UAS391 and TCH1516 exhibited single nucleotide polymorphisms (SNPs) (Additional file 1: Table S1), of which 47 mapped within open reading frames (ORFs) and 5 within intergenic regions.

Based on genome sequence data and comparison of whole genome maps (Fig. 1), FPR3757 is closely related to TCH1516 [24], and thus also to UAS391, and forms less biofilms in the dynamic biofilm model, compared to UAS391 $(P=0.0172)$ and similar to TCH1516 (Fig. 2) $(P=1.0000)$. As the complete sequence for FPR3757 is also available, we then compared UAS391 and FPR3757 and identified a total of 91 gene loci where UAS391 and FPR3757 exhibited SNPs (Additional file 2: Table S2), of which 60 mapped within ORFs and 31 within intergenic regions. To further narrow down this initial list of deviating genes, we identified an overlap between the gene alleles of UAS391, TCH1516 and FPR3757 (Additional file 1: Table S1 and Additional file 2: Table S2). This reduced the initial list of genetic variations between strains UAS391 and TCH1516 to only those SNPs that were identical in the two weak biofilm forming strains TCH1516 and FPR3757 but different in UAS391, potentially identifying those SNPs primarily responsible for the increased biofilm formation in UAS391. This comparison yielded 18 SNPs as shown in Table 2. Of this, SNPs in transposases and phage-elements (USA300HOU_0286 and 1488) were excluded from further analysis $(n=2)$.

Similarly, comparative genome alignment was performed for TCH1516, FPR3757 and UAS391 to confirm the data obtained by whole genome sequencing (Additional file 3: Figure S1).

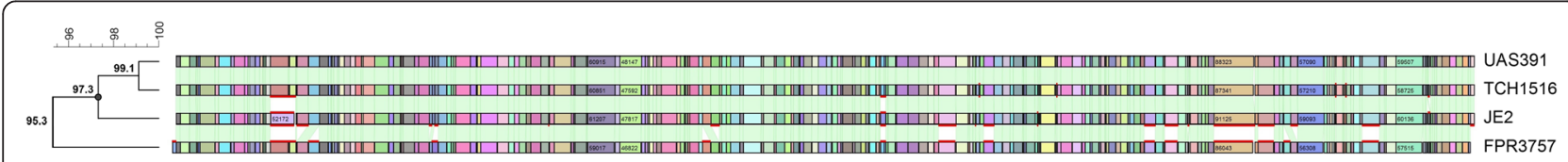

Fig. 1 Whole genome maps of USA300 strains. Green lines indicate identity of restriction pattern among the maps and red horizontal marks represent the variations 


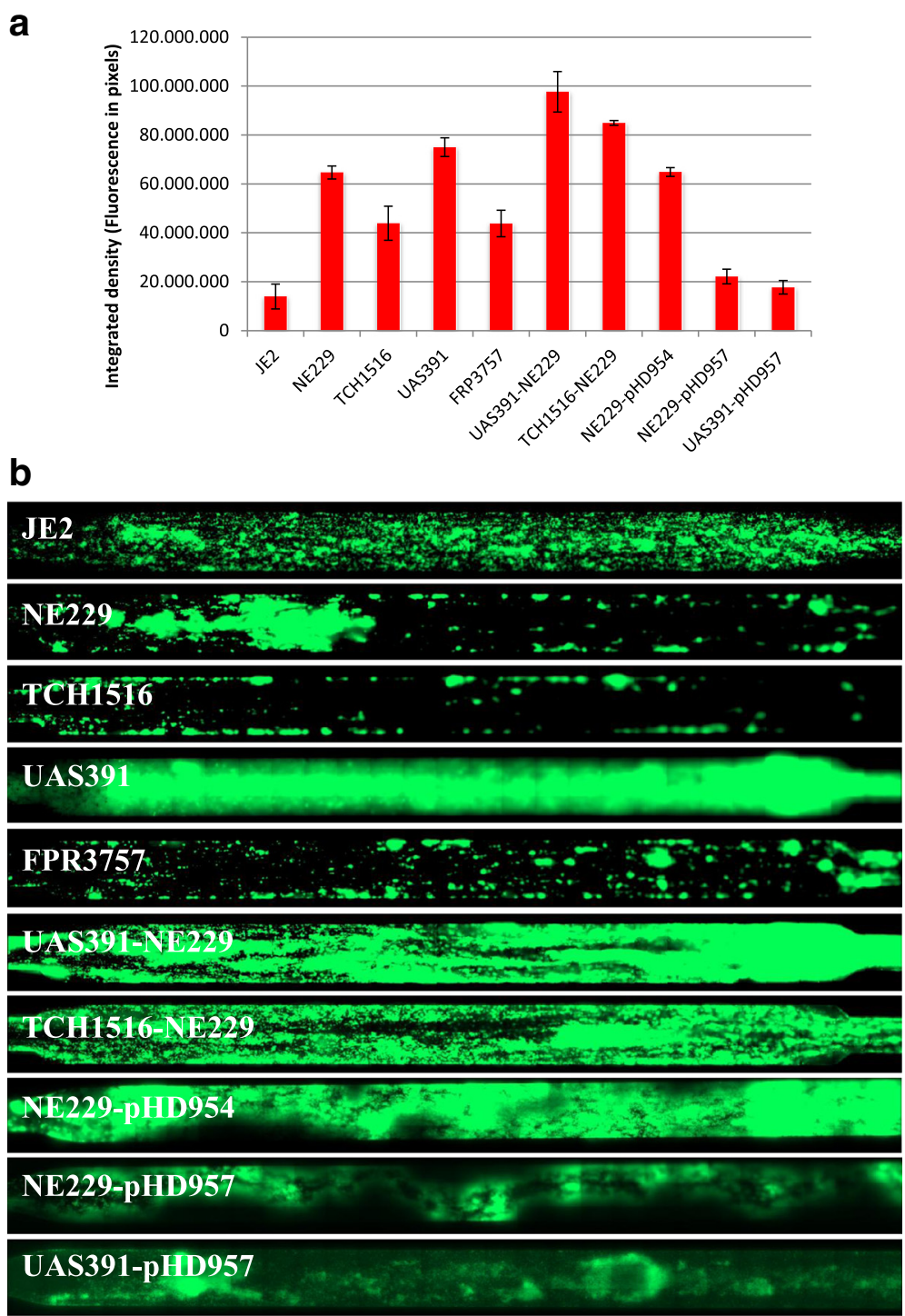

Fig. 2 Biofilm formation of USA300 strains in the dynamic shear flow assay. Quantification (a) and visualization (b) of biofilms formed by wild-type USA300 strains and corresponding derivate strains

Identification of genes involved in biofilm formation using gene knockout mutants

In order to investigate the role of the corresponding genes in biofilm formation, we obtained 12 mutants from the Network on Antimicrobial Resistance in S. aureus (NARSA) library in which transposon insertions map in genes affected by SNPs, belonging to the above identified group of 16 genes enumerated in Table 2. For the remaining four candidate genes, USA300HOU_0521, 1051, 1166, and 0502, corresponding knockout mutants were not present in the library and these were not studied further. We also randomly selected five genes affected by SNPs solely in either TCH1516 or FPR3757 in comparison to UAS391. In total, the 17 mutants obtained from the NARSA library (corresponding to USA300HOU_0953, 0155, 1162, 1260, 1372, 2197, 2626, 2631, 2641, 2319, 2602, 2678, 2654, 1626, 2026, $1338,1943)$ were phenotypically tested in duplicate for their ability to form flow biofilms using the library parental strain JE2 as the corresponding control. Significant positive and negative variations of the degree of biofilm formation as compared to JE2 were found $(P=0.0018$, ANOVA, $\mathrm{F}=4.44, \mathrm{df}=17)$. Out of the 17 mutants tested (Fig. 3), one mutant NE229 showed a 2-fold increase in biofilm formation compared to JE2 $(P=0.0024)$. NE229 harbours the transposon insertion at position 393 in 
Table 2 Single nucleotide polymorphisms (SNPs) between TCH1516/FPR3757 and UAS391. Consensus base represents TCH1516 and FPR3757, allele represents a corresponding base in UAS391. Predicted amino acid changes in UAS391 compared to TCH1516 and FPR3757 were not displayed for synonymous SNPs

\begin{tabular}{|c|c|c|c|c|}
\hline $\begin{array}{l}\text { Consensus base } \\
\text { (TCH1516/FPR3757) }\end{array}$ & $\begin{array}{l}\text { Allele } \\
\text { UAS391 }\end{array}$ & $\begin{array}{l}\text { Gene locus tag } \\
\text { (in TCH1516/FPR3757) }\end{array}$ & $\begin{array}{l}\text { Predicted amino acid change } \\
\text { (TCH1516/FPR3757) }\end{array}$ & Gene and putative function \\
\hline G & $\mathrm{T}$ & $\begin{array}{l}\text { USA300HOU_0286/ } \\
\text { SAUSA300_0267 }\end{array}$ & Leu1Phe & IS1272 transposase \\
\hline $\mathrm{T}$ & C & $\begin{array}{l}\text { USA300HOU_0155/ } \\
\text { SAUSA300_0145 }\end{array}$ & & $\begin{array}{l}\text { Phosphate-import protein PhnD/ABC Transporter, } \\
\text { periplasmic binding protein }\end{array}$ \\
\hline G & A & $\begin{array}{l}\text { USA300HOU_0521/ } \\
\text { SAUSA300_0513 }\end{array}$ & Met386lle & gt/X: glutamyl-tRNA synthetase \\
\hline A & C & $\begin{array}{l}\text { USA300HOU_1051/ } \\
\text { SAUSA300_1013 }\end{array}$ & Asn267Thr/Asn263Thr & Bacterial cell division membrane protein FtsW \\
\hline A & G & $\begin{array}{l}\text { USA300HOU_1162/ } \\
\text { SAUSA300_1119 }\end{array}$ & Glu524Gly & fakA: Fatty acid kinase \\
\hline G & T & $\begin{array}{l}\text { USA300HOU_1166/ } \\
\text { SAUSA300_1123 }\end{array}$ & Gly169Val & $\begin{array}{l}\text { fabD: malonyl CoA-acyl carrier protein transacylase } \\
\text { (Lipid metabolism) }\end{array}$ \\
\hline A & G & $\begin{array}{l}\text { USA300HOU_1372/ } \\
\text { SAUSA300_1327 }\end{array}$ & Val1114Ala & Ebh: Cell Wall-Associated Fibronectin-Binding Protein \\
\hline C & A & $\begin{array}{l}\text { USA300HOU_2197/ } \\
\text { SAUSA300_2161 }\end{array}$ & & $\begin{array}{l}\text { Hyaluronate lyase is a glycosaminoglycan (GAG) } \\
\text { polysaccharide lyase family. This family consists of a } \\
\text { group of secreted bacterial lyase enzymes capable of } \\
\text { acting on glycosaminoglycans, such as hyaluronan and } \\
\text { chondroitin, in the extracellular matrix of host tissues, } \\
\text { contributing to the invasive capacity of the pathogen. }\end{array}$ \\
\hline G & A & $\begin{array}{l}\text { USA300HOU_2631/ } \\
\text { SAUSA300_2566 }\end{array}$ & Pro27Ser & $\begin{array}{l}\text { HTH-type Transcriptional regulator ArcR, signal } \\
\text { transduction system }\end{array}$ \\
\hline C & T & $\begin{array}{l}\text { USA300HOU_2641/ } \\
\text { SAUSA300_2576 }\end{array}$ & & $\begin{array}{l}\text { PTS system, fructose-specific II ABC component, multi } \\
\text { protein system involved in regulation of metabolic and } \\
\text { transcriptional process }\end{array}$ \\
\hline $\mathrm{T}$ & C & $\begin{array}{l}\text { USA300HOU_2319/ } \\
\text { SAUSA300_2285 }\end{array}$ & & Aldose 1-epimerase \\
\hline G & A & $\begin{array}{l}\text { USA300HOU_2602/ } \\
\text { SAUSA300_2542 }\end{array}$ & $\operatorname{Arg} 517^{* 1}$ & $\begin{array}{l}\text { Acyl-coenzyme A synthetases/AMP-(fatty) acid ligases } \\
\text { (Lipid metabolism) }\end{array}$ \\
\hline A & C & $\begin{array}{l}\text { USA300HOU_2654/ } \\
\text { SAUSA300_2589 }\end{array}$ & Ser1782Ala & $\begin{array}{l}\text { Serine-rich adhesin for platelets, cell surface protein } \\
\text { precursor; KxYKXGKXXW signal peptide }\end{array}$ \\
\hline C & T & $\begin{array}{l}\text { USA300HOU_0502/ } \\
\text { SAUSA300_0486 }\end{array}$ & Thr85Met & Uncharacterized protein YabR \\
\hline C & T & $\begin{array}{l}\text { USA300HOU_1626/ } \\
\text { SAUSA300_1585 }\end{array}$ & Ser35Leu & $\begin{array}{l}\text { tRNA threonylcarbamoyladenosine dehydratase (Cells } \\
\text { lacking this gene have a normal growth phenotype, } \\
\text { but are unable to survive in a competitive growth } \\
\text { situation with the wild-type strain. They display only } \\
\text { the t6A but not the ct6A modification in tRNAs, and } \\
\text { have lower decoding efficiency than wild-type. They } \\
\text { show no defects in antibiotic sensitivity. In growth } \\
\text { competition experiments, a tcdA mutant shows reduced } \\
\text { fitness compared to wild-type, but outcompetes a } \\
\text { csdA mutant.) }\end{array}$ \\
\hline A & G & $\begin{array}{l}\text { USA300HOU_2026/ } \\
\text { SAUSA300_1984 }\end{array}$ & Lys118Arg & Putative membrane peptidase YdiL \\
\hline $\mathrm{T}$ & C & $\begin{array}{l}\text { USA300HOU_1488/ } \\
\text { SAUSA300_1436 }\end{array}$ & & phage lipoprotein \\
\hline $\mathrm{T}$ & - & $\begin{array}{l}\text { USA300HOU_1338/ } \\
\text { SAUSA300_1298 }\end{array}$ & Phe130fs $^{2}$ & 5-bromo-4-chloroindolyl phosphate hydrolysis protein \\
\hline
\end{tabular}




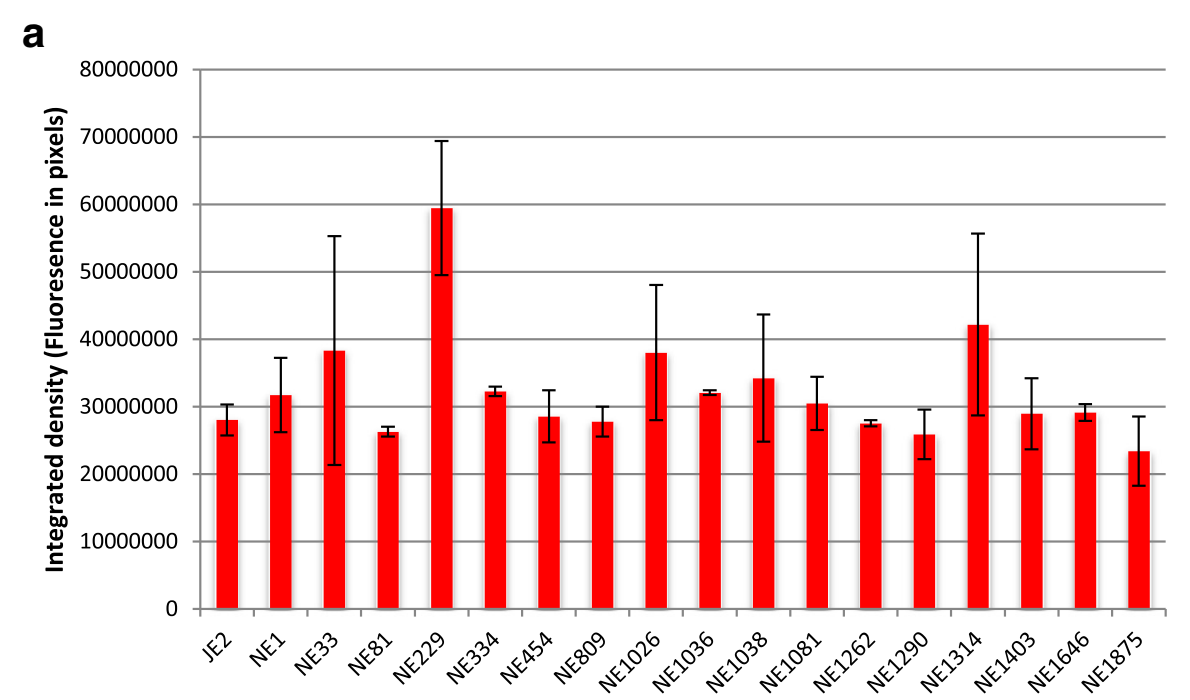

b
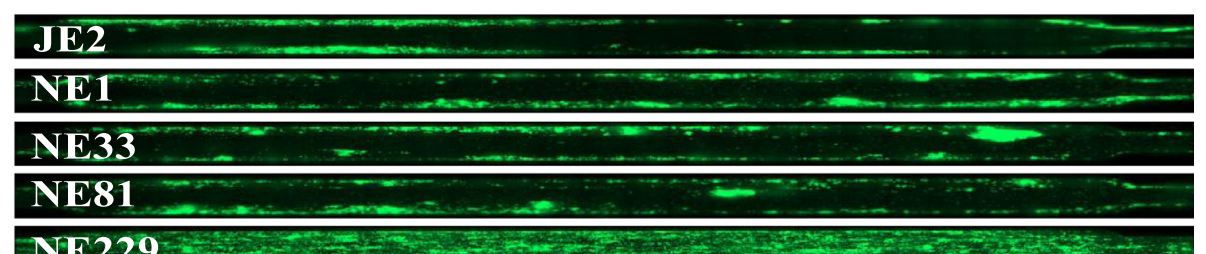

NE229

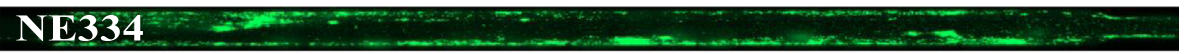

NE454

NE809

NE1026 .

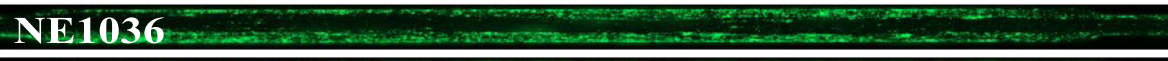

NE1038

NE1262
NE1290
NE1314
NE1403
NE1646
NE1875

Fig. 3 Biofilm formation of USA300 strains in the dynamic shear flow assay. Quantification (a) and visualization (b) of biofilms formed by JE2 and its transposon mutants

ORF SAUSA300_1119 encoding the fatty acid kinase fakA located in the genome from position 1223940 to 1225586 nt [25] (Additional file 4: Figure S2).

Reverse transcription PCR (RT-PCR) was performed to determine whether the bursa aurealis insertion had affected transcription of SAUSA300_1119. Primers were designed downstream of the transposon mutation. Transcription of SAUSA300_1119 in NE229 showed a significant decrease compared to that in wild-type strains FPR3757 and JE2 (Cts of 31.9, 26.2, and 26.0, respectively) $(P<0.0001$, ANOVA, $F=1599.54, \mathrm{df}=6$ ). Similar decrease in transcription was also observed for the TCH1516-EryS and the UAS391-EryS strain transduced with the SAUSA300_1119 mutated allele from NE229 $(P<0.0001)$ (Fig. 4).

The SAUSA300_1119 in UAS391 harbours a non-synonymous SNP (Glu524Gly) as compared to strains 


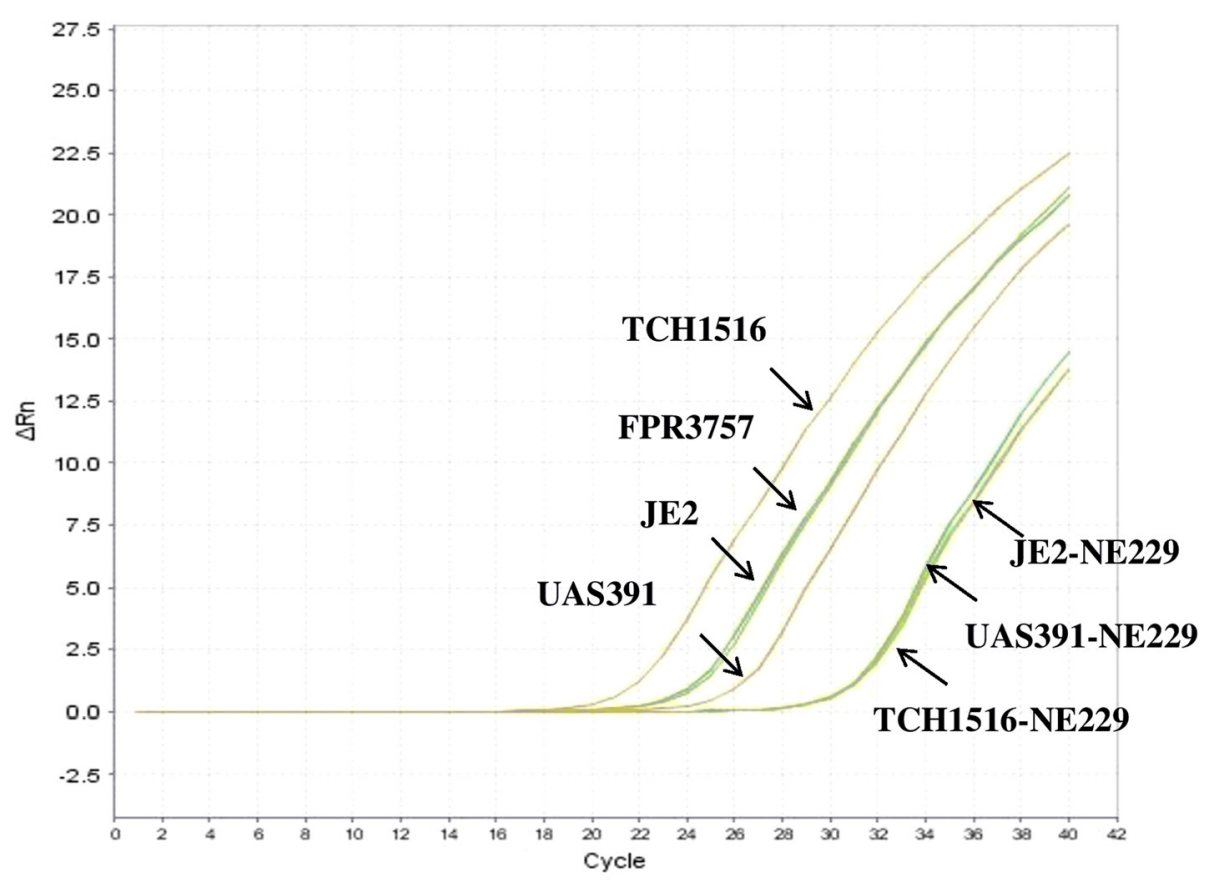

\begin{tabular}{|c|c|c|}
\hline Strain & Ct (me an) & Ct (Std Dev) \\
\hline JE2 & 26.0482 & 0.1228 \\
\hline FPR3757 & 26.229 & 0.0322 \\
\hline UAS391 & 27.8113 & 0.0867 \\
\hline TCH1516 & 23.6982 & 0.0541 \\
\hline JE2-NE229 & 31.8522 & 0.4254 \\
\hline TCH1516-NE229 & 31.5482 & 0.054 \\
\hline UAS391-NE229 & 32.2225 & 0.2792 \\
\hline
\end{tabular}

Fig. 4 Transcription levels of the fakA gene in UAS391, TCH1516, FPR3757, JE2 and in corresponding transductant strains. Transcription levels of fakA were measured in duplicate by real-time RT-PCR and expressed as an amplification plot and Ct mean values

TCH1516 and FPR3757 (Table 2). BLAST search identified it as a unique SNP and it can thus be considered as the mutant version of the corresponding gene present in TCH1516 and FPR3757, with similar wild-type alleles being present among other sequenced $S$. aureus isolates (Additional file 4: Figure S2).

\section{Transductants mutated in fakA gene exhibit elevated biofilm formation}

In order to further corroborate the negative role of $f a k A$ in biofilm formation by USA300, the knockout mutation in gene SAUSA300_1119 was transferred from NE229 into both UAS391-EryS and TCH1516-EryS employing phage-mediated transduction resulting in UAS391NE229 and TCH1516-NE229, respectively. Knocking out SAUSA300_1119 in TCH1516 (locus tag USA300HOU_1162) resulted in a 1.7 -fold increase in biofilm formation compared to the wild type TCH1516
$(P=0.0007)$, and at levels similar to the wild-type UAS391 $(P=0.9710)$ (Fig. 2). This effect was also observed in the UAS391 background where UAS391-NE229 exhibited even more abundant biofilm formation than the wild-type UAS391 ( $P=0.0510)$ (Fig. 2).

\section{A plasmid-borne copy of the wild-type fakA gene complements the mutant phenotype}

In order to unambiguously prove that the enhanced biofilm phenotype observed in the NE229 strain is indeed due to the knockout mutation of the fakA gene, the mutant strain was complemented with a plasmid-borne intact fakA allele present in the TCH1516 strain or with the corresponding SNP-containing allele present in the UAS391 strain, yielding NE229-pHD957 and NE229pHD954 strains, respectively. As shown in Fig. 2, the amount of biofilm produced by NE229-pHD957 was 2.9-fold lower as compared to NE229 $(P=0.0003)$, and 
similar to the amount of biofilm produced by the parent JE2 strain $(P=0.9831)$, whereas the amount of biofilm produced by NE229-pHD954, containing only mutant fakA allele was the same as in NE229 $(P=1.0000)$. Additionally, complementing UAS391 with pHD957 resulted in a 4.3-fold decrease in biofilm mass as compared to the parent UAS391 $(P<0.0001)$.

\section{Discussion}

By comparing the whole genome sequences of closely related USA300 strains that strongly differed in their capacity to form biofilms in a dynamic flow model, identified genetic differences (SNPs) were hypothesized to be responsible for this altered biofilm phenotype. Seventeen transposon mutants knocked out in these genes in the USA300-JE2 background (NARSA strains) were evaluated for the degree of biofilm formation in comparison to the parent JE2. Transposon-mediated interruption of one of the tested divergent genes, fakA [25], previously known as $v f r B$ [26], resulted in a 4.6-fold increase in biofilm formation as compared to the parental control strain JE2. In the other USA300 strains, UAS391 and TCH1516, the fakA::Tn mutations also led to increased biofilm formation, and further complementation experiments confirmed the role of $f a k A$ in the regulation of biofilm formation.

Running the protein sequence of SAUSA300_1119 in Simple Modular Architecture Research Tool (SMART) identified two domains within the protein sequence: Dak2 encoding the predicted phosphatase domain of the dihydroxyacetone kinase family (35 to $200 \mathrm{nt}$ ), and Dak1_2 encoding the kinase domain of the dihydroxyacetone kinase family (236 nt to $548 \mathrm{nt}$ ). The glutamic acid to glycine change is located at position 524 of the protein corresponding to its kinase domain. These proteins, collectively called Dak2 domain proteins have homologues in a wide variety of bacteria. Transposon insertions in $f a k A$ were first isolated in a large $S$. aureus transposon (bursa aurealis-bearing) insertion mutants library screen based on an increased resistance to an antimicrobial peptide [27]. Importantly, this $f a k A$ (then called $d a k 2$ ) mutant exhibited an altered membrane phospholipid composition compared to its wild-type parent [27]. Recent studies have further delineated the multiple functions of $f a k A$ as an important regulator of virulence factors [26] and as a fatty acid kinase responsible for host fatty acid incorporation by $S$. aureus [25]. Interestingly, in the present study, complementation by the fakA allele of TCH1516, which is the consensus allele, in the fakA::Tn NE229 and in the UAS391 strains decreased the amount of biofilm formation by 2.7 - and 4.3 -fold, respectively, to the level observed in the JE2 wild-type strain. However, similar complementation by the UAS391 fakA allele, which harbours the glutamic acid to glycine change, did not affect biofilm formation in fakA::Tn NE229. Taken together, these results imply that the mutation in the UAS391 fakA might have impacted the catalytic activity of the kinase, and whether UAS391 exhibits an altered membrane phospholipid composition due to this amino acid change, which increased its biofilm forming ability, remains to be studied.

Interestingly, microarray-based gene expression data from a USA300 fakA::Tn mutant showed significant up-regulation of 26 and down-regulation of 19 genes [25]. The downregulated genes included $s a e P$ and $s a e Q$ that are part of the saeRS two-component regulatory system and are known to regulate the activity of the saeS-encoded sensor histidine kinase [28]. The saeRS system was also recently shown to be a negative regulator of biofilm formation in S. aureus [29]. This study showed that a $S$. aureus Newman $\triangle$ saeRS strain exhibited an enhanced biofilm phenotype, similar to the fakA::Tn JE2-NE229 mutant and the UAS391 wild-type strains in our study. Taken together, these phenotypic and gene expression data strongly suggest a potential interaction between $f a k A$ and saeRS in negatively regulating biofilm formation in $S$. aureus. Finally, mice infected with vfrB::Tn (fakA::Tn) S. aureus have been shown to develop significantly larger abscess areas and dermonecrosis [26], which also reflects the increased biofilm abundance observed for the fakA::Tn mutant in our study.

Thus utilizing a combination of functional assays and genomics, we identified $f a k A$, a known virulence factor regulator and a fatty acid kinase, as an important negative regulator of biofilm formation in S. aureus USA300.

\section{Conclusions}

The sequential approach used here, starting from comparing clonally related (as per optical mapping) clinical isolates with different clinically relevant phenotypes, then comparing the respective total genome information allowed to pinpoint a gene locus, that is clearly of relevance for biofilm formation in S. aureus.

\section{Additional files}

Additional file 1: Table S1. Complete list of SNPs in genes representing divergent alleles in UAS391 and TCH1516 and their putative gene functions. TCH1516 was used as a reference for comparison with the allelic change in UAS391. Nucleotide changes in bold red represent the common SNPs in both FPR3757 and TCH1516 as compared to UAS391 (also in Table 2). (XLSX 14 kb)

Additional file 2: Table S2. Complete list of SNPs in genes representing divergent alleles in UAS391 and FPR3757 and their putative gene functions. FPR3757 was used as a reference for comparison with the allelic change in UAS391. Nucleotide changes in bold red represent the common SNPs in both FPR3757 and TCH1516 as compared to UAS391 (also in Table 2). (XLSX 14 kb)

Additional file 3: Figure S1. Comparative genome alignment of USA300, TCH1516, FPR3757 and UAS391 shows high conservation 
between these genomes. Pink regions show homology and the vertical lines shows conserved blocks. The UAS391 13 kb genomic region (from $680369 \mathrm{bp}$ to $693620 \mathrm{bp}$ ) is translocated to (1630711 to $1642611 \mathrm{bp}$ ) in FPR3757. (PDF $116 \mathrm{~kb}$ )

Additional file 4: Figure S2. Multiple alignment of fakA gene sequences in Staphylococcus aureus and Staphylococcus epidermidis. Unique nucleotide variation in SAUSA300_1119 of UAS391 encoding a fatty acid kinase fakA at position $1223940 \mathrm{nt}$, corresponding to nt 393 of the gene. The respective SAUSA300_1119 allele in UAS391 contains a non-synonymous SNP (Glu to Gly) as compared to strains TCH1516 and FPR3757. The Genbank accession numbers were indicated among these 50 strains. The first 45 strains represent $S$. aureus, the last 5 strains represent S. epidermidis. (PDF $897 \mathrm{~kb}$ )

\section{Abbreviations}

BHI: Brain heart infusion broth medium; BLAST: Basic local alignment search tool; CA: Community-acquired; Dak2: Dihydroxyacetone kinase 2; fakA: Fatty acid kinase A; HA: Hospital-acquired; HA-MRSA: Hospital-acquired methicillin-resistant Staphylococcus aureus; LB: Lysogeny broth medium; MRSA: Methicillin-resistant Staphylococcus aureus; NARSA: Network on Antimicrobial Resistance in Staphylococcus aureus; ORF: Open reading frame; PIA: Polysaccharide intercellular adhesion; PNAG: Polymeric N-acetylglucosamine; RT-PCR: Reverse transcription polymerase chain reaction; SMART: simple modular architecture research tool; SNP: Single nucleotide polymorphism; Tn: Transposon; UPGMA: Unweighted-pair group method with arithmetic averages; vfrB: Virulence factor regulator B; WGM: Whole genome mapping.

\section{Competing interests}

The authors declare that they have no competing interests.

\section{Authors' contributions}

SMK, HG and JS conceived the study. JS, SDB, PM, JPH, HDG and ATR carried out the experimental work. SMK, HDG, JPH, HG and JS drafted the manuscript. BBX performed bioinformatic analysis. All authors read and approved the final manuscript.

\section{Authors' information}

Not applicable

\section{Availability of data and materials}

Not applicable

\section{Acknowledgments}

We thank the Network on Antimicrobial Resistance in Staphylococcus aureus (NARSA) Program for providing bursa aurealis transposon mutants and other S. aureus strains. We also thank Olivier Denis (Université Libre de Bruxelles) for providing strains and Francine Deboeck for excellent technical assistance. This work is supported by funding from Research Foundation Flanders (FWO G.0983.10 N and Research grant no. 1522412 N). B.B.X. is supported by University of Antwerp Research Funds (BOF-DOCPRO 2012-27450).

\section{Funding}

This work is supported by funding from Research Foundation Flanders (FWO G.0983.10N and Research grant no. 1522412N). B.B.X. is supported by University of Antwerp Research Funds (BOF-DOCPRO 2012-27450). SDB is supported by a research grant by the Institute for the Promotion of Innovation by Science and Technology (IVT) in Flanders, project number 140746.

\footnotetext{
Author details

'Laboratory of Medical Microbiology, Vaccine and Infectious Disease Institute, University of Antwerp, Antwerp, Belgium. ${ }^{2}$ Viral Genetics Research Group, Vrije Universiteit Brussel, Brussels, Belgium. ${ }^{3}$ Structural Biology Brussels, Vrije Universiteit Brussel, Brussels, Belgium. ${ }^{4}$ Structural and Molecular Microbiology, Structural Biology Research Center, VIB, Brussels, Belgium. ${ }^{5}$ Department of Medical Microbiology, Campus Drie Eiken, University of Antwerp, S6, Universiteitsplein 1, B-2610 Wilrijk, Belgium.
}

Received: 22 May 2015 Accepted: 25 September 2015 Published online: 26 October 2015

\section{References}

1. Stefani S, Varaldo PE. Epidemiology of methicillin-resistant staphylococci in Europe. Clin Microbiol Infect. 2003;9:1179-86.

2. Jones SM, Morgan M, Humphrey TJ, Lappin-scott H. Effect of vancomycin and rifampicin on meticillin-resistant Staphylococcus aureus biofilms. Lancet. 2001;357:40-1.

3. Raad I, Alrahwan A, Rolston K. Staphylococcus epidermidis : emerging resistance and need for alternative agents. Clin Infect Dis. 1998;26:1182-7.

4. Donlan RM, Costerton JW. Biofilms: survival mechanisms of clinically relevant microorganisms. Clin Microbiol Rev. 2002;15:167-93.

5. Resch A, Rosenstein R, Nerz C, Go F. Differential gene expression profiling of Staphylococcus aureus cultivated under biofilm and planktonic conditions. Appl Environ Microbiol. 2005;71:2663-76.

6. Resch A, Leicht S, Saric M, Pásztor L, Jakob A, Götz F, et al. Comparative proteome analysis of Staphylococcus aureus biofilm and planktonic cells and correlation with transcriptome profiling. Proteomics. 2006;6:1867-77.

7. Vanhommerig E, Moons P, Pirici D, Lammens C, Hernalsteens J-P, De Greve $\mathrm{H}$, et al. Comparison of biofilm formation between major clonal lineages of methicillin resistant Staphylococcus aureus. PLoS ONE. 2014;9, e104561.

8. Fey PD, Endres JL, Yajjala K, Widhelm TJ, Boissy RJ, Bose JL, et al. A genetic resource for rapid and comprehensive phenotype screening of nonessential Staphylococcus aureus genes. MBio. 2013;4:1-8.

9. Fitzgerald JR. Methicillin Resistant Staphylococcus Aureus (MRSA): Protocols. Totoway: Humana Press; 2007. p. 103-12.

10. Adams MH. Bacteriophages. New York: Interscience; 1959. p. 1-620.

11. Bateman BT, Donegan NP, Jarry TM, Palma M, Cheung AL. Evaluation of a tetracycline-inducible promoter in Staphylococcus aureus in vitro and in vivo and its application in demonstrating the role of sigB in microcolony formation. Infect Immun. 2001;69:7851-7.

12. Kreiswirth BN, Lofdahl S, Betley MJ, O'Reilly M, Schlievert PM, Bergdoll MS, et al. The toxic shock syndrome exotoxin structural gene is not detectably transmitted by a prrophage. Nature. 1983;305:709-12.

13. Sabirova JS, Xavier BB, leven M, Goossens H, Malhotra-Kumar S. Whole genome mapping as a fast-track tool to assess genomic stability of sequenced Staphylococcus aureus strains. BMC Res Notes. 2014;7:704.

14. Sabirova J, Xavier BB, Lammens C, Goossens H, Malhotra-Kumar S. Transcriptomics profile of biofilms produced by Staphylococcus aureus USA300. Intersci Conf Antimicrob Agents Chemother 2013, Oral presentation.

15. Zerbino DR, Birney E. Velvet: algorithms for de novo short read assembly using de Bruijn graphs. Genome Res. 2008;18:821-9.

16. Bankevich A, Nurk S, Antipov D, Gurevich AA, Dvorkin M, Kulikov AS, et al. SPAdes: a new genome assembly algorithm and its applications to single-cell sequencing. J Comput Biol. 2012;19:455-77.

17. Darling ACE, Mau B, Blattner FR, Perna NT. Mauve: multiple alignment of conserved genomic sequence with rearrangements. 2004:1394-1403.

18. Sabirova J, Xavier BB, Hernalsteens JP, De Greve H, Malhotra-Kumar S, Goossens H. Complete Genome sequences of two prolific biofilm-forming Staphylococcus aureus isolates belonging to USA300 and EMRSA-15. Genome Announc. 2014;2:2-3.

19. Tamura K, Stecher G, Peterson D, Filipski A, Kumar S, Arabia S. MEGA6 : Molecular Evolutionary Genetics Analysis version 6. 0. Mol Biol Evol. 2013;30:2725-9.

20. Benoit MR, Conant CG, lonescu-Zanetti C, Schwartz M, Matin A. New device for high-throughput viability screening of flow biofilms. Appl Environ Microbiol. 2010;76:4136-42.

21. Highlander SK, Hultén KG, Qin X, Jiang H, Yerrapragada S, Mason EO, et al. Subtle genetic changes enhance virulence of methicillin resistant and sensitive Staphylococcus aureus. BMC Microbiol. 2007;7:99.

22. Shukla SK, Pantrangi M, Stahl B, Briska AM, Stemper ME, Wagner TK, et al. Comparative whole-genome mapping to determine Staphylococcus aureus genome size, virulence motifs, and clonality. J Clin Microbiol. 2012;50:3526-33.

23. Tewhey R, Cannavino CR, Leake JAD, Bansal V, Topol EJ, Torkamani A, et al. Genetic structure of community acquired methicillin-resistant Staphylococcus aureus USA300. BMC Genomics. 2012;13:508.

24. Diep BA, Gill SR, Chang RF, Phan TH, Chen JH, Davidson MG, et al. Complete genome sequence of USA300, an epidemic clone of community-acquired meticillin-resistant Staphylococcus aureus. Lancet. 2006;367:731-9.

25. Parsons JB, Broussard TC, Bose JL, Rosch JW, Jackson P, Subramanian C, et al. Identification of a two-component fatty acid kinase responsible for host fatty acid incorporation by Staphylococcus aureus. Proc Natl Acad Sci U S A. 2014;111:10532-7. 
26. Bose JL, Daly SM, Hall PR, Bayles KW. Identification of the Staphylococcus aureus vfrAB operon, a novel virulence factor regulatory locus. Infect Immun. 2014;82:1813-22.

27. Li M, Rigby K, Lai Y, Nair V, Peschel A, Schittek B, et al. Staphylococcus aureus mutant screen reveals interaction of the human antimicrobial peptide dermcidin with membrane phospholipids. Antimicrob Agents Chemother. 2009;53:4200-10.

28. Jeong D, Hoonsik C, Jones MB, Shatzkes $K$, Sun F, Ji Q, et al. The auxiliary protein complex SaePQ activates the phosphatase activity of sensor kinase SaeS in the SaeRS two-component system of Staphylococcus aureus. Mol Microbiol. 2012;86:331-48

29. Cue D, Junecko JM, Lei MG, Blevins JS, Smeltzer MS, Lee CY. SaeRS-dependent inhibition of biofilm formation in Staphylococcus aureus Newman. PLOS ONE. 2015;10, e0123027.

\section{Submit your next manuscript to BioMed Central and take full advantage of:}

- Convenient online submission

- Thorough peer review

- No space constraints or color figure charges

- Immediate publication on acceptance

- Inclusion in PubMed, CAS, Scopus and Google Scholar

- Research which is freely available for redistribution 